\title{
Assessment and tuning of the behaviour of a microphysical characterisation scheme
}

\author{
M. Galletti ${ }^{1,2}$, P. P. Alberoni ${ }^{1}$, V. Levizzani ${ }^{3}$, and M. Celano ${ }^{1,4}$ \\ ${ }^{1}$ A.R.P.A. Emilia Romagna, Servizio Idrometeorologico, Bologna, Italy \\ ${ }^{2}$ TU Chemnitz, Fakultät Elektrotechnik und Informationstechnik, Chemnitz, Germany \\ ${ }^{3}$ ISAC-CNR, Bologna, Italy \\ ${ }^{4}$ Università degli studi di Ferrara, Dip.di Fisica, Ferrara, Italy
}

Received: 25 October 2004 - Revised: 18 April 2005 - Accepted: 19 April 2005 - Published: 13 May 2005

\begin{abstract}
The correct classification of prevailing bulk hydrometeor type within a radar resolution volume is a challenge task even if a full set of polarimetric radar observables is available. Indeed scattering and propagation effects from the variety of hydrometeors present interact each others and sometimes, if not often, tend to obscure the characteristic signature of each weather radar target type. This consideration is enforced when the atmospheric volume is sampled with a wavelength where both Mie scattering effects and attenuation start to become relevant.

In this paper, we utilize the hydrometeor classification scheme developed at the National Severe Storms Laboratory (USA). Briefly, the scheme uses a fuzzy logic approach to combine different polarimetric variables and environmental temperature in order to determine the most likely type of prevalent hydrometeor in the radar volume. This means that the resulting classification is based on two characteristics: the volume polarimetric responses and the thermal value. The relative balance between these two is managed through the coefficients in the fuzzy scheme. We have observed that these parameters are crucial in order to get "physical reasonable result", independently from the meteorological character of the event investigated.

Our work is based on a reduced set of polarimetric variables $\left(Z\right.$ and $\left.Z_{D R}\right)$ as input. Data used in this study were collected by a $C$-band radar over weather events ranging from convective to stratiform.
\end{abstract}

\section{Introduction}

Considering the scientific evidence accumulated over the last decade, radar meteorologists made it clear that polarimetry will play a fundamental role in the forthcoming use of weather radars. Polarimetric radar data have actually potential applications to many diverse fields from improving

Correspondence to: P. P. Alberoni

(palberoni@smr.arpa.emr.it) quantitative precipitation estimates to hydrometeors identification, up-to improving initial conditions and constraints for short term forecast numerical models. Zrnic and Ryzhkov (1999) give a summary of the state-of-the-art of polarimetry for weather surveillance radar.

A never-ending task such as the best estimation of the rain pattern will benefit from polarimetric information. Indeed, the correct classification of the prevailing bulk hydrometeor type within a radar resolution volume should be a prerequisite of any quantitative rain-retrieving scheme. Further, the classification is also useful for warning purposes and for validation of non-hydrostatic models where the physical parameterizations are so advanced that major hydrometeor species are represented.

Most of the work in this field is based on the use of Sband data, but the progressive deployment of C-band polarimetric radars in Europe motivate the research community to increase work at shorter wavelengths. Radar data taken at C-band or X-band are affected by attenuation and resonance (see among others May et al., 1999; Zrnic et al., 2000). The difficulties in identifying the bulk hydrometeor types within a radar resolution volume are amplified by propagation effects at C-band. We refer chiefly to the difficulties of interpreter the joint occurrences in radar signatures of ambiguities in hydrometeor classes, occurrence of artifacts in the data, and uncertainties in radar calibration.

For this reason particular attention and efforts should be addressed to adapt procedures from S-band and validate these at C-band. In this paper, we use the scheme developed at the National Severe Storms Laboratory (hereinafter referred to as NSSL scheme), with a reduced set of polarimetric variables $\left(Z, Z_{D R}\right)$ collected at $\mathrm{C}$-band.

\section{Hydrometeors classification scheme}

The NSSL scheme is based on fuzzy logic and the reader should refer to Straka et al. (2000) and Zrnic et al. (2001) for an exhaustive introduction to it. In brief, let us say that it 


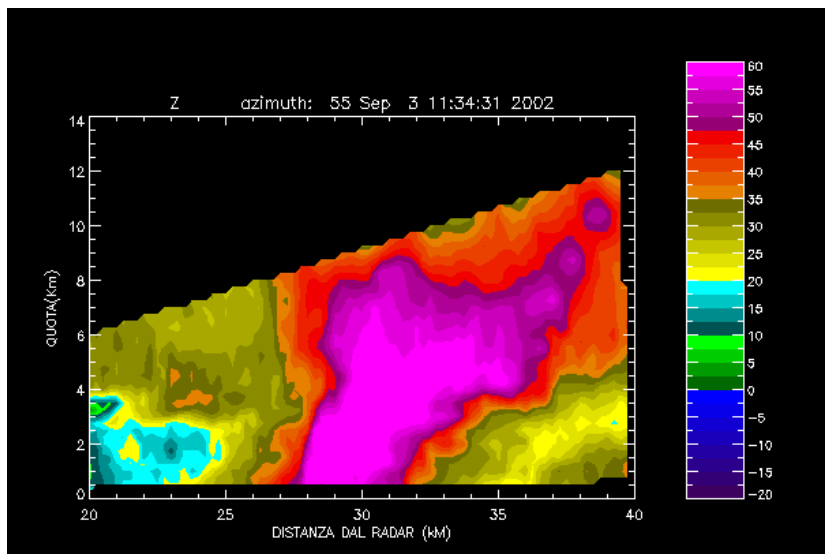

Fig. 1. Reflectivity of the convective event observed by the SPC radar (3 September 2002).

is based on a combination of weighting (membership) functions associated with a particular hydrometeor type. These are two-dimensional functions, the first argument being one of the polarimetric variables and the other the equivalent reflectivity factor $Z$. The classification is accomplished by taking the highest value of the combination of weighting functions for each image pixel.

The original version of the NSSL scheme uses four different radar variables plus the temperature profile (to remove certain ambiguities). The variables are: equivalent reflectivity factor $(Z)$, differential reflectivity $\left(Z_{D R}\right)$, specific differential phase (KDP) and the cross-correlation coefficient between horizontally and vertically polarized waves $(\rho h v)$.

The NSSL scheme is designed to take advantage of the presence of all available polarimetric variables and also to work with a reduced set of these. As discussed in Zrnic et al. (2001), the most discriminating variables are the reflectivity factor and the differential reflectivity. Based on this fact, we expect to obtain reasonable results with the San Pietro Capofiume C-band polarimetric radar (SPC hereafter), managed by ARPA Emilia Romagna, which provides the two most significant variables for the classification.

The environmental temperature is the other parameter used for the classification. The algorithm uses a standard atmospheric profile of $6.5^{\circ} \mathrm{km}^{-1}$ starting from the surface temperature measured by ground local stations.

With such a reduced set we are able to study how sensitive the algorithm is to the possible set of the parameters used and to their interrelationship.

Ten hydrometeor classes can be discriminated by the algorithm: light rain (LR), moderate rain (MR), heavy rain (HR), rain dominated by large drops (LD), rain/hail mixture (RH), graupel and/or small hail (GSH), hail (HA), dry snow (DS), wet snow (WS) and ice crystals (HIC).

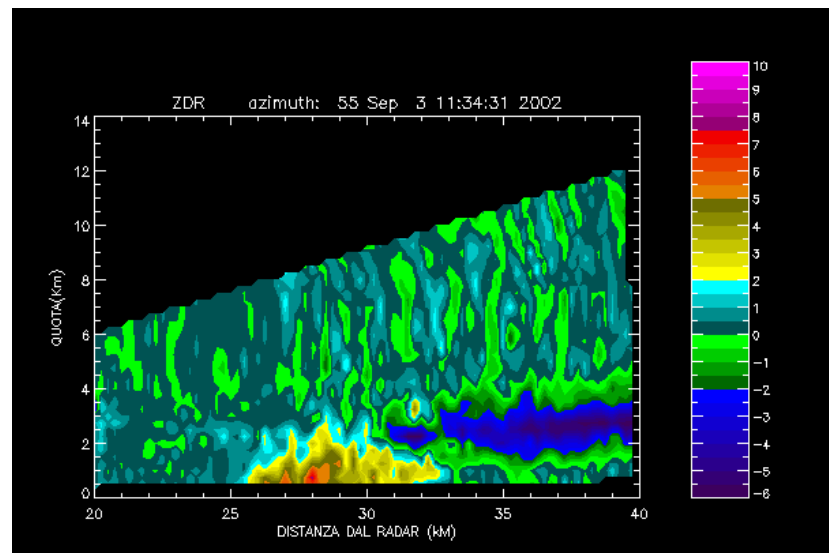

Fig. 2. Differential reflectivity of the convective event observed by the SPC radar.

\section{Overall description of the behaviour}

The application of the NSSL scheme with a reduced set of variables lead us to reformulated it in way to highlight the contribution from the polarimetric observables and from the environmental temperature:

$S=\frac{A_{\text {rpol }} \cdot W_{\text {rpol }}+A_{t} \cdot W_{t}}{W_{\text {rpol }}+W_{t}}$

where $A_{\text {rpol }}$ and $A_{t}$ are the results obtained from the polarimetric and the thermal membership functions respectively and $W_{\text {rpol }}$ and $W_{t}$ are the "weight" coefficients introduced to account for the different influence of the terms that come into play.

Respect to the original scheme, the implementation has preserved the choice of setting to one the term $W_{r p o l}$ and had to test the algorithm sensitivity to different values of $W_{t}$.

The algorithm assigns to each hydrometeor class a number that is representative of its "probability of presence". Each resolution volume will be assigned to the class that, for that cell, shows the highest value.

To shield us from unwanted misclassification due to noise, no-data circumstances or some kind of artifacts, it is wise to introduce a threshold value on the result $S$ (refer to Eq. 1) that cuts off the assignment to hydrometeor types. Thus the classification is done by taking the highest value of the combination only if is greater than the threshold value $S$.

In order to assess the sensitivity of the scheme to small, if not infinitesimal, variations of the temperature weight $W_{t}$ and the threshold value $S$, we consider a "representative" convective event. The reflectivity and differential reflectivity are displayed in Fig. 1 and Fig. 2.

A direct application of the classification scheme with the values of $S=0.5$ and $W_{t}=1.0$ reproduces well known problems (see Fig. 3) already documented (e.g., among others, in Alberoni et al., 2002):

- The attenuation cone behind the main convective core is filled with hail; 


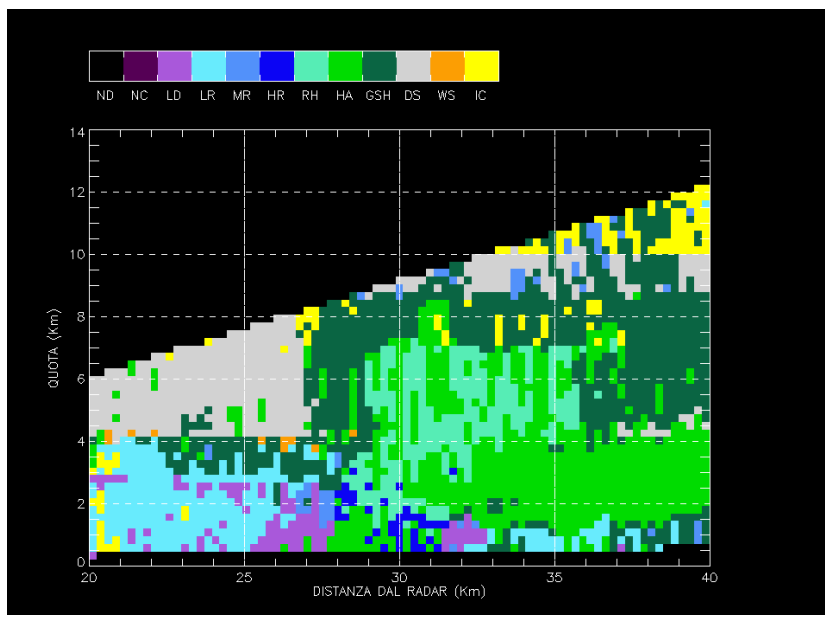

Fig. 3. Hydrometeor classification for the data in Fig. 1 and Fig. 2 $\left(S=0.5, W_{t}=1.0\right)$.

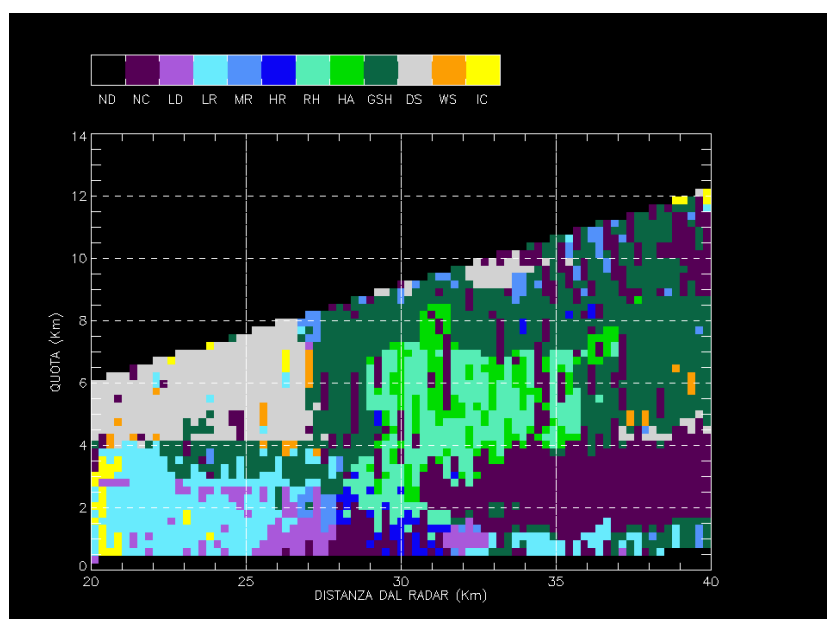

Fig. 4. Hydrometeor classification for the data in Fig. 1 and Fig. 2 ( $S=0.5, W_{t}=0.8$ ).

Table 1. Results of the classification scheme with different values of the driving parameters.

\begin{tabular}{lll}
\hline & $S$ & $W_{t}$ \\
\hline Reasonable & 0.48 & 0.92 \\
SI & 0.48 & 0.93 \\
Reasonable & 0.46 & 0.85 \\
SI & 0.46 & 0.86 \\
\hline
\end{tabular}

- Too many ice crystals identified at the highest levels.

These are, of course, not realistic in terms of cloud microphysics. We refer to this type of hydrometeors classification as "spurious image" (SI).

Further, some pixels are assigned to hydrometeor classes that are in contrast with the environmental temperature. In-

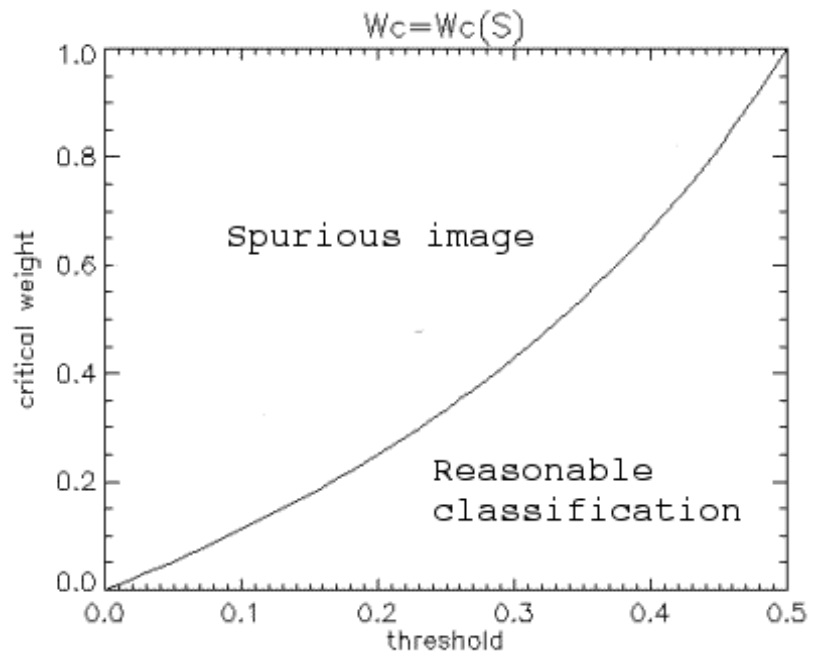

Fig. 5. Boundary of the transition between the SI configuration (upper part of the diagram) and a reasonable classification. The curved boundary represents the variation of the critical temperature weight as a function of the acceptance threshold.

deed, we note the presence of ice crystals close to the ground and moderate rain at roughly $8 \mathrm{~km}$ above ground.

A decreasing of the temperature weight causes a transition in the behavior of the algorithm. The attenuated area is now unclassified and also the ice crystal pixels have diminished (see Fig. 4). Note that the pixels which were wrongly classified are now unclassified. On the same stream, also the area close to the base of the convective core is now partially classified as LD and partially unclassified.

This transition is very sharp and its effect does not increase even when the temperature weight decreases to very low values ( $W_{t}=0.4$ or lower). If the weight value gets too low the algorithm looses its thermal characterization with obvious negative impact on the classification itself. In this case the classification that has lost the thermal characterization, this means that hydrometeor appear where they should not be present (e.g. snow close to ground during summertime at sea level) We will refer to the behavior as Thermal effect (TE).

These sensitivities and behaviors of the classification algorithm do not depend on the specific image under consideration: similar images for the same couples of $S$ and $W_{t}$, were reproduced for convective and stratiform events. This suggests that these observed characteristics are intrinsic properties of the scheme.

\section{Analysis of the transition to SI type}

From an analysis of the images produced using a variety of couples of the "driving parameters" it is clear that the transition between a reasonable classification and a classification with the SI characteristics takes place over a well defined sharp boundary. Examples of such a boundary are the couples in Table 1. 


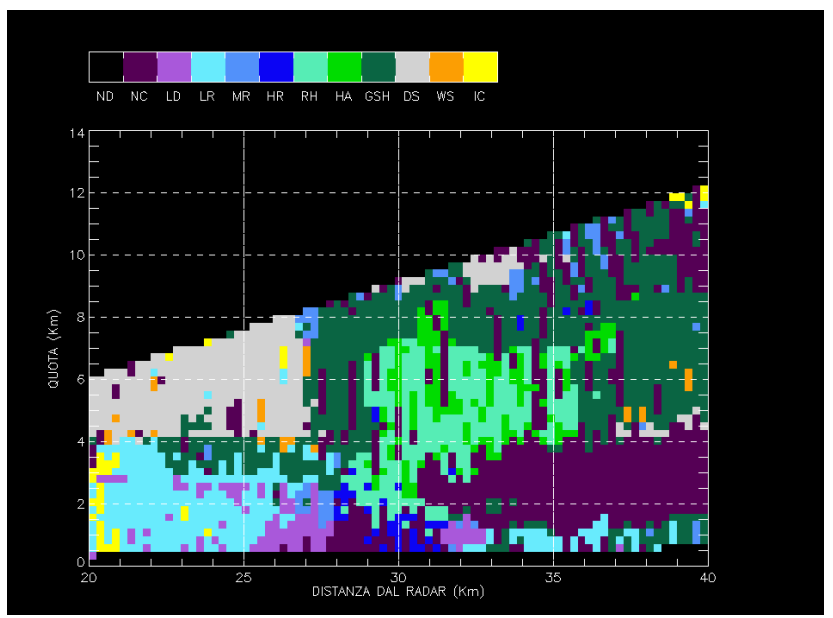

Fig. 6a. Hydrometeor classification for the data in Fig. 1 and Fig. 2 $\left(S=0.55, W_{t}=0.81\right)$.

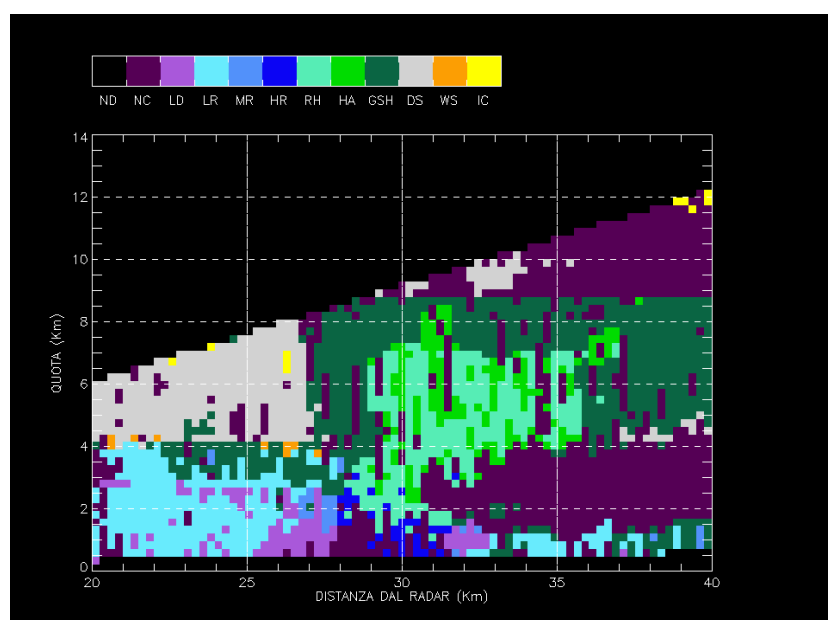

Fig. 6b. Hydrometeor classification for the data in Fig. 1 and Fig. 2 $\left(S=0.55, W_{t}=0.82\right)$.

A close look to the algorithm reveals that the temperature shape functions are such that for each temperature there is at least one hydrometeor type that could exist in such environment.

As a direct consequence of this constraint there is always some hydrometeor type that gains the maximum score from the thermal component of the algorithm. If in such cases the acceptance threshold, which is the combination between the thermal and the polarimetric components of the algorithm, is lower than the thermal one, we will accept as classified some pixels without any polarimetric agreement. This means that, for example, if $W_{t}=1$ then we should have $S>0.5$.

Such behavior could be deducted from the mathematical expression of the algorithm. In this configuration the acceptance threshold could be simplified as:

$S>\frac{k_{2} \cdot W_{t}}{1+W_{t}}$

where $k_{2}$ is the value of the temperature shape function.

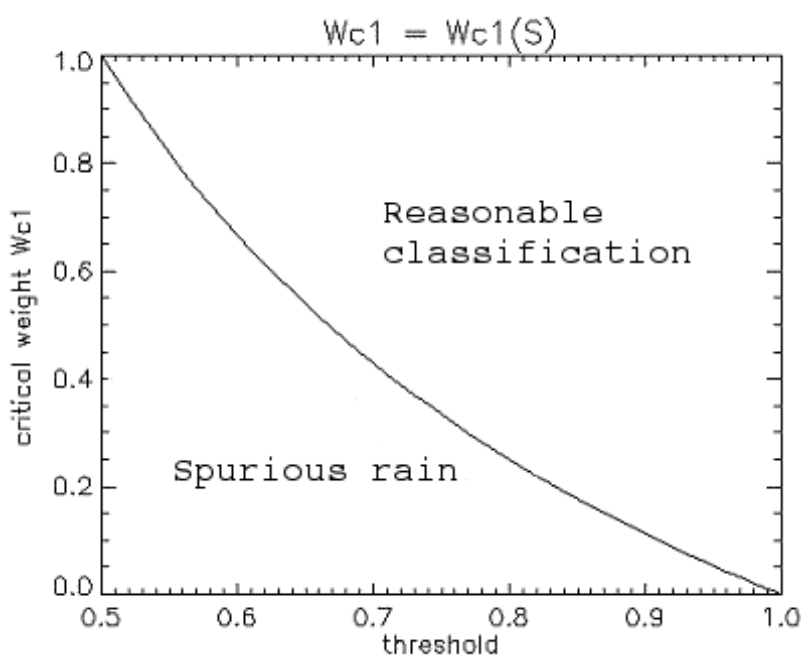

Fig. 7. Boundary of the transition between the TE configuration (lower part of the diagram) and a reasonable classification. The line represents the variation of the critical temperature weight as a function of the acceptance threshold.

In the range of valid temperature $[-40,+40]^{\circ} \mathrm{C}$ we can invert this equation to extract a function that describes the variation of the critical temperature weight as a function of the selected acceptance threshold:

$W c=\frac{S}{1-S}$

This function is displayed in Fig. 5 .

\section{Analysis of the transition to TE type}

From the analysis of the images examined so far it can be stated that the TE configuration is always present if the acceptance threshold is below 0.5 regardless of the thermal weight and disappears if we choose a couple like $S=0.51$ and $W_{t}=1.0$.

We need to understand the basic rules of such behavior and further to analyze the TE transition for values of acceptance threshold above 0.5 .

For this reason we focused our attention on a convective case using different couples of parameters. As for the SI transition the TE transition happens across a very sharp boundary. Some examples of such transition are displayed in Figs. 6a and b. This effect is clearly visible if we analyse carefully the maps. Pixels classified as rain are present at the top of the storm well above the melting layer $(8 \mathrm{~km}$ and more, Fig. 6a), similarly ice crystal are classified in the first $4 \mathrm{~km}$ in the left part of Fig. 6a), both problems disappear after the transition (Fig. 6b). In this case the relevant pixels are not classified.

In a broad sense the TE configuration is the analog of the SI configuration. In such case some hydrometeors are classified only on the base of the polarimetric component without any contribution from the thermal component. As for the SI 

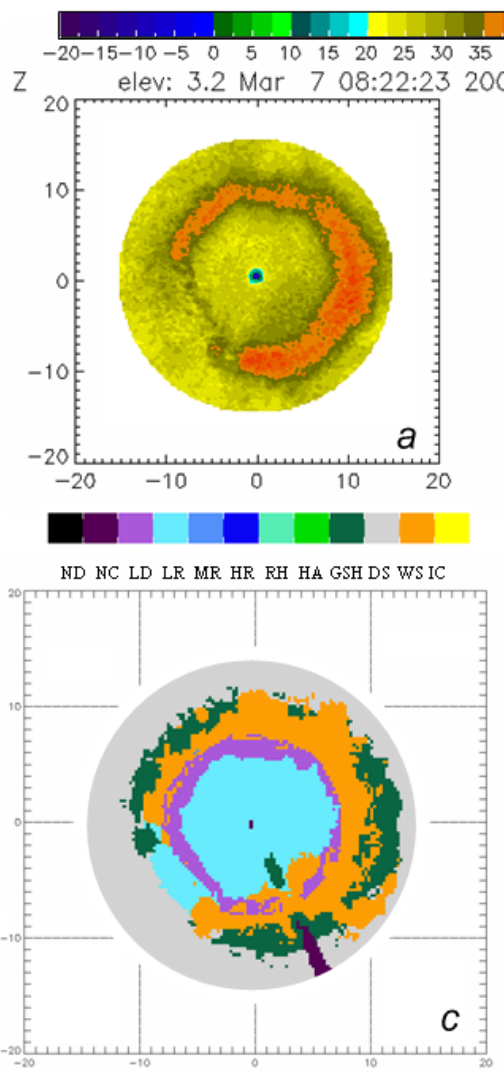
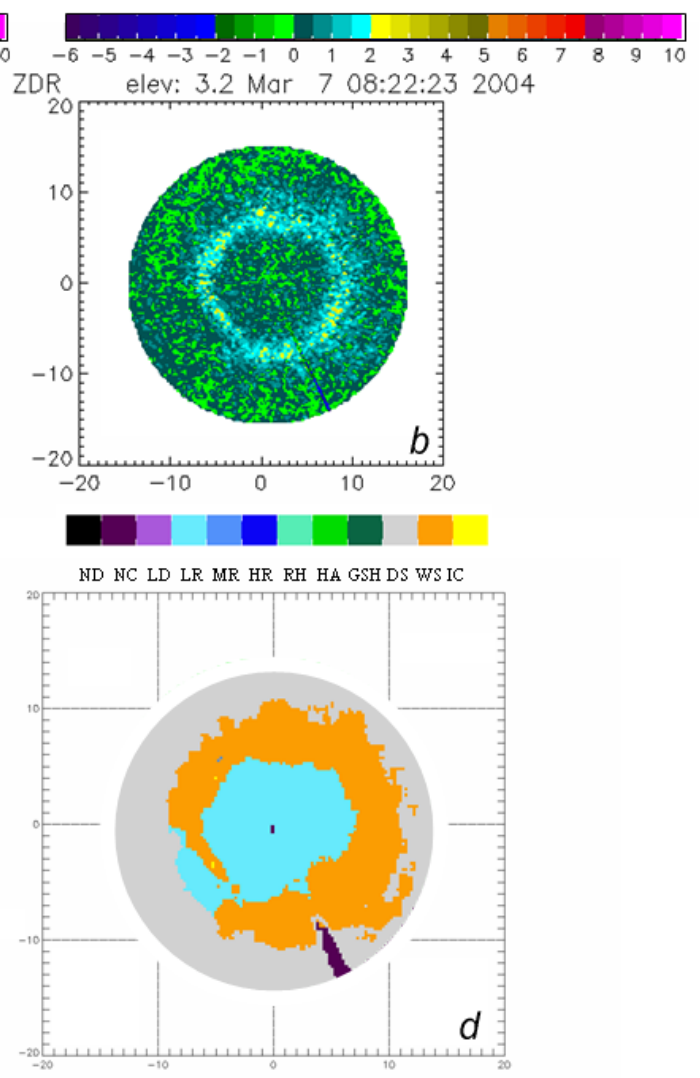

Fig. 8. Stratiform event of 07-03-2004. Three degree elevation ppi of (a) horizontal reflectivity ZH, (b) differential reflectivity ZDR, (c) SPC hydrometeor classification, (d) SPC hydrometeor classification, considering the second identified class for graupel and large drops.

case we have to draw upon the mathematical formulation of the algorithm and impose the acceptance threshold be greater than the single polarimetric component. The equation is thus:

$S>\frac{k_{1}}{1+W_{t}}$

where $k_{1}$ is the value of the polarimetric shape function.

If we invert the equation, we obtain the variation of the critical weight as a function of the acceptance threshold for the TE transition (Fig. 7).

\section{Application of $\mathrm{HC}$ scheme to a snowfall event}

On 7 March 2004, a conspicuous snowfall interested the area explored by the SPC radar. This event has been selected as a representative one for a stratiform precipitation.

The presence of a bright band signature is clearly highlighted in the PPI shown in Figs. 8a, b. In spite of this radar observation, the Borgo Panigale (Bo) synoptic station (roughly $20 \mathrm{~km}$ south of the radar) reports the presence of snow during the whole day, even if the surface temperature measured by Saiarino station (roughly $10 \mathrm{~km}$ south-east of the radar site) indicated a big variability of temperature ranging from 0 to $5^{\circ} \mathrm{C}$ during the day (at 8.22 , time of data shown in Fig. 8 , the temperature is $2.8^{\circ} \mathrm{C}$ ). It is possible that the region around the radar was interested by a rain-snow mixture event at the lowest layers or by a double bright band event, with a second bright band closer the ground.

A direct application of the NSSL scheme is shown in Fig. 8c, where the classification indicates the presence of light rain in the area close the ground, wet snow in the melting layer region and dry snow in the region above the bright band.

The reader should note the presence of graupel and large drops near the bright band, which is completely unrealistic for a stratiform event. The reason of this misclassification is due to the practical impossibility to introduce the real temperature field in the classification algorithm, further the limited set of radar variables used causes that for some hydrometeor classes the output of the classification algorithm is not able to resolve ambiguities in classification, defining a single suitable hydrometeor class.

This is exact the case which occurs in proximity of the bright band level. In this particular situation, analyzing the second identified hydrometeor class, is possible to note that those pixels classified by the algorithm as graupel are indeed wet snow in the inner edge or dry snow in the outer edge for the region misclassified. Similarly the LD area is mainly re-classified as wet snow.

The result of this reclassification using the second choice particles is shown in Fig. 8d. These hydrometeors are much more realistic for a stratiform event. 


\section{Conclusion}

The classification scheme has proved to be robust enough to be used with a reduced set of polarimetric variables provided a careful choice of the parameters is done, actually values of $S=0.55$ and $W_{t}=1.0$ are used in the operational classification.

In this configuration a reasonable classification will be obtained. As a counter effect the number of unclassified pixels will increase as the threshold value increases. It is clear that the correct choice of parameters depends on the type of application and is in the hand of the analyst.

Any sensitivity to the parameters could be avoided by combining the terms with products of them that automatically guarantee the simultaneous contribution of all the terms to the overall acceptance value, even if this approach presents some disadvantages too (for more details see the work of Liu and Chandrasekar, 2000). The multiplicative approach, however, has been less investigated in the literature and operational implementations in the US make use of sums rather than products.

Acknowledgements. This work is partially supported by CARPE DIEM, a research project supported by the European Commission under the 5th FP (Contract No. EVG1-CT-2001-0045), by the INTERREG IIIB CADSES "RISK AWARE" (3B064) project, and form the GNDCI through the project RAM.

Edited by: L. Ferraris

Reviewed by: anonymous referees

\section{References}

Alberoni, P. P., Zrnic, D. S., Ryzhkov, A. V., and Guerrieri, L.: Use of a fuzzy logic classification scheme with a C-band polarimetric radar: first results, Proceedings of the Second European Conference on Radar Meteorology (ERAD) in conjunction with COST 717 mid-term seminar, Delft, Netherlands, 18-22 November 2002, pp. 324-327, 2002.

Liu, H. and Chandrasekar, V.: Classification of Hydrometeors Based on Polarimetric Radar Measurements: Development of Fuzzy Logic and Neuro-Fuzzy Systems, and In Situ Verification, J. Atmos. Oceanic Technol., 17, 140-164, 2000.

May, P. T., Keenan, T. D., Zrnic, D. S., Carey, L. D., and Rutledge, S. A.: Polarimetric radar measurements of tropical rain at a $5 \mathrm{~cm}$ wavelength, J. Appl. Meteor., 38, 750-765, 1999.

Straka, J. M., Zrnic, D. S., and Ryzhkov, A. V.: Bulk hydrometeor classification and quantification using polarimetric radar data: synthesis of relations, J. Appl. Meteor., 39, 1341-1372, 2000.

Zrnic, D. S. and Ryzhkov, A. V.: Polarimetry for weather surveillance radars, Bull. Amer. Meteor. Soc., 80, 389-406, 1999.

Zrnic, D. S., Keenan, T. D., Carey, L. D., and May, P. T.: Sensitivity analysis of polarimetric variables at a $5-\mathrm{cm}$ wavelengthn rain, J. Appl. Meteor., 39, 1514-1526, 2000.

Zrnic, D. S., Ryzhkov, A. V., Straka, J. M., Liu, Y., and Vivekanandan, J.: Testing a procedure for automatic classification of hydrometeor types, J. Atmos. Oceanic Technol., 18, 892-913, 2001. 